SCIENTIFIC REPORT

\title{
Clinical and ultrastructural findings in mare's tail lines of the corneal epithelium
}

\author{
S Akhtar, A J Bron, K M Meek, L W Voon
}

Br J Ophthalmol 2004;88:864-867. doi: 10.1136/bjo.2003.033456

\begin{abstract}
Aims: Mare's tail lines are uncommon, grey, parallel, tapering epithelial lines, which may give rise to discomfort or to visual symptoms. The authors report the clinical and ultrastructural findings in two patients

Methods: Therapeutic debridement was performed in two patients suffering from mare's tail disorder. The loosely attached epithelium was removed and fixed in glutaraldehyde containing cuprolinic blue and processed in a standard fashion for electron microscopy.

Results: Ultrastructural studies showed an avascular, collagenous pannus extending under the whole of the excised epithelium in each case and separated from it by a thickened basal lamina like zone. The basal laminar material extended into the epithelium in folds, in keeping with the clinical features. Interesting ultrastructural features included: (1) a lack of hemidesmosomes in relation to the basal laminar material; (2) trapping of degenerate keratocytes within the invaginating basal laminar folds; (3) an unusual regularity and fine dimensions of the collagen fibrils and proteoglycans making up the subepithelial pannus. The basal laminar material contained proteoglycans and small fibres similar in appearance to long spacing collagen.

Conclusions: Mare's tail lines are caused by basal laminar material, assumed to be chiefly of epithelial origin, which invaginates the corneal epithelium. The presence of occasional keratocytes within the invaginating folds suggests that there may be a stromal contribution to the disorder. The visibility of mare's tail lines in the focal beam of the slit lamp is likely to be related to the thickness and light scattering properties of the invaginations and of the subepithelial deposits. Tractional forces, imposed by lid action, could explain their horizontal disposition.
\end{abstract}

$\mathrm{T}$ he epithelial basement membrane disorders (EMBD) have been described under different names in the past, such as moonscapes, ${ }^{1}$ fingerprint lines, ${ }^{2}$ epithelial microcystic dystrophy, ${ }^{3}$ superficial corneal disorder, ${ }^{5}$ and map dot fingerprint dystrophy. ${ }^{6}{ }^{7}$ Additional features of these conditions include bleb like disorder and mare's tail lines. At light microscopic level, fingerprint lines consist of concentric, ridge like epithelial inclusions which enter at a normal to the surface and extend parallel to the surface of the cornea. ${ }^{8}{ }^{9}$ In map dot fingerprint dystrophy, basal laminar invaginations, similarly disposed, give rise to the maps, which are a chief component of the disorder. Collections of degenerate epithelial cells, forming the characteristic, white "Cogan's" microcysts, are sequestered behind these map like invaginations. Although there is some evidence for inheritance of the basement membrane disorders, ${ }^{10}$ this is not well established, and it may be more appropriate to speak of map dot fingerprint disorder.
Brown and Bron $(1976)^{5}$ discussed the clinical features of various epithelial corneal lines, including mare's tail lines, which are the subject of this paper. Here we report the clinical and ultrastructural findings in two patients with this disorder.

\section{METHODS}

Patient A was a 74 year old female referred by her general practitioner with left recurrent corneal erosion following a past abrasion. Best corrected visual acuity was $20 / 20$ in the right eye and 20/30 in the left eye. She was noted to have mare's tail lines on both corneas and was treated with tear substitutes and a bandage contact lens. She subsequently developed right herpes zoster ophthalmicus with stromal keratitis, which resolved after topical treatment. Because of a persistent visual disturbance in the right eye with surface epithelial changes, she underwent right corneal debridement under local anaesthesia. Postoperatively, the eye remained comfortable and a vision of 20/30 was achieved.

Patient B was a 61 year old female referred by her optician with bilateral corneal opacities and reduced visual acuity. Best corrected visual acuity was 20/40 in each eye and she was diagnosed as having bilateral mare's tail lines. Visual acuity in the left eye deteriorated to 20/60 six months after presentation and she underwent left corneal debridement under local anaesthesia. Postoperatively, the left acuity improved to $20 / 30$.

Therapeutic debridement was performed in each patient. The epithelium was loosely attached to Bowman's layer and was removed as a continuous epithelial sheet.

The epithelium tissue was fixed in $2.5 \%$ glutaraldehyde containing $0.05 \%$ cuprolinic blue (BDH Ltd, Dorset, UK) in a critical electrolyte concentration mode, then dehydrated through a graded ethanol series and embedded in Spurr resin. Semithin $(1 \mu \mathrm{m})$ and ultrathin $(70 \mathrm{~nm})$ sections were cut from three blocks from each patient. Semithin sections were stained with toluidine blue for light microscopy, and ultrathin sections were stained with uranyl acetate and lead citrate and observed by transmission electron microscopy (JEOL 1010; JEOL Ltd, Akishima, Japan).

\section{RESULTS}

The typical clinical appearance of mare's tail dystrophy in patient A is shown in figure 1. Clinical results: mare's tail lines were, bilateral, faint, white, horizontally disposed, elevated, concentric tapering lines, within the corneal epithelium, which may give rise to visual symptoms and discomfort and are treatable by epithelial debridement. In both patients, light microscopy showed a thick epithelium overlying a greatly thickened basement membrane, forming invaginating folds into the epithelium (figs 2A-D). In patient A, the basement membrane material formed broad extensions

Abbreviations: $B M$, basement membrane; $E M B D$, epithelial basement membrane disorder. 
occupying about a quarter of the epithelial thickness, displacing the basal epithelial cells towards the surface wing cells, and occupied one quarter of the epithelial thickness (fig 2A and $\mathrm{B}$ ). In patient $\mathrm{B}$, the basement membrane material invaginations formed loop like folds extending to within a few cells of the surface of the basal epithelial cells, and extended into the upper layer of wing cells.

Ultrastructural findings were similar in each patient and characterised by a thickened, subepithelial basement membrane associated with the invaginating intraepithelial folds. Within the subepithelial, basement membrane like material, there were large amounts of proteoglycans, collagen fibres (fig 3A), and small fibrils resembling long spacing collagen (fig 3B). In some parts, the epithelium was elevated by mounds of basement membrane material (fig 3C), intermixed with proteoglycans and degenerate collagen fibres (fig 3D). In neither patient were hemidesmosomes identified, either along the basal cell-basal/basal laminar interface, or in relation to the basal laminar folds.

The subepithelial deposits were multilamellar, consisting of irregularly alternating layers of collagen fibres and basement membrane like material. There were large, scattered empty spaces below the basal epithelial cells and a subjacent, avascular, collagenous pannus mixed with basement membrane material. More deeply, there were pools of proteoglycans and microfibrillar material lying between collagen fibres (fig 3E). Patches resembling Bowman's layer were also present (fig 3F).

The invaginating intraepithelial sheets consisted of folds of basement membrane like material, containing collagen fibres and proteoglycans (fig 4A). Figure 4B shows the apex of an invaginating fold containing a keratocyte with a collection of collagen, sandwiched in the mid zone (fig 4A and C).

In both patients, basal epithelial cells in the neighbourhood of the invaginations showed electron dense cytoplasm, apoptotic nuclei, and prominent nuclear blebbing (fig 5A and $\mathrm{B})$. The nuclei were surrounded by perinuclear vacuoles containing dense material (fig 5C and D). The vacuoles associated with the nuclei of the basal cells were very large compared to those of the wing cells. The cytoplasm of individual cells contained large numbers of tonofilaments around vacuoles and nuclei. The intercellular spaces, away
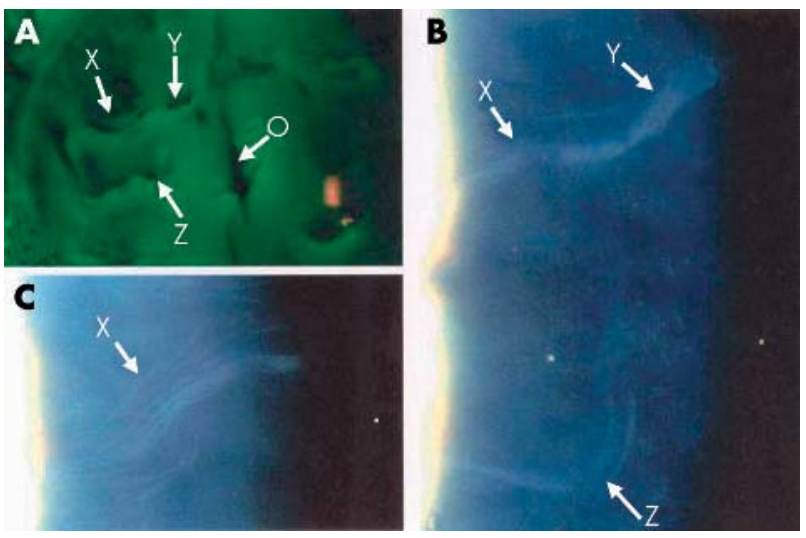

Figure 1 (A) Distribution of mare's tail lines in the right eye of patient $A$ shown with fluorescein in the tear film. Multiple groups of mare's tail lines $(X, Y, Z)$ are seen, showing the typical "handle bar moustache" configuration, made up of single or double crescents, convex downwards. The mare's tail elevations appear dark within the fluorescent film. Oblique streaks $(\mathrm{O})$ are artefacts produced by random break up of the tear film. (B) High power view of two groups of mare's tail lines ( $X$ and $Y$ ) (shown in $A$ ) by broad, oblique slit lamp illumination (C) High power view of the group labelled $X$, emphasising the fibrillar appearance of the lines.
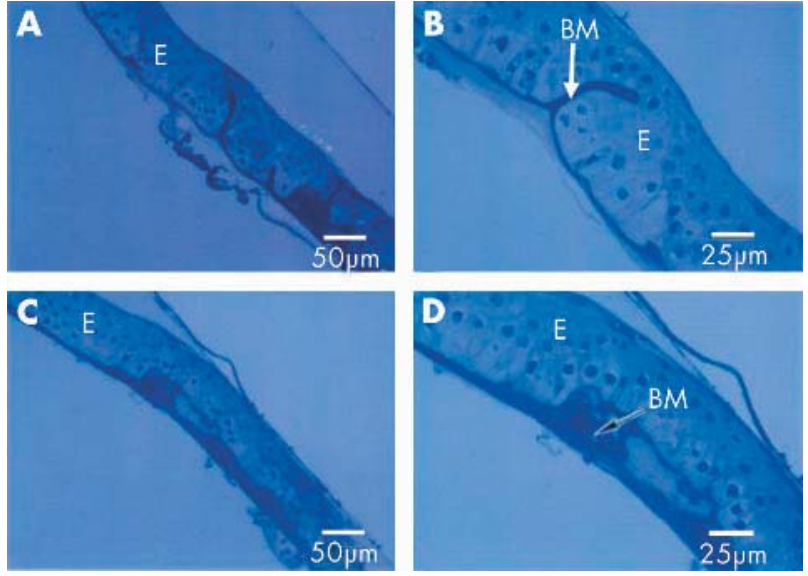

Figure 2 Light micrograph of epithelium (E) affected by mare's tail lines. (A and B) Invaginating folds of basement membrane material (BM). (C and D) Accumulation of basement membrane material (BM).

from the invaginating material, were prominent, although desmosomes were well developed (fig 5D).

\section{DISCUSSION}

Mare's tail lines are faint, grey, horizontally disposed, elevated, intraepithelial lines, arranged in parallel bunches and with tapering at their ends. Their appearance has been likened to that of a mare's tail, but the frequently upward
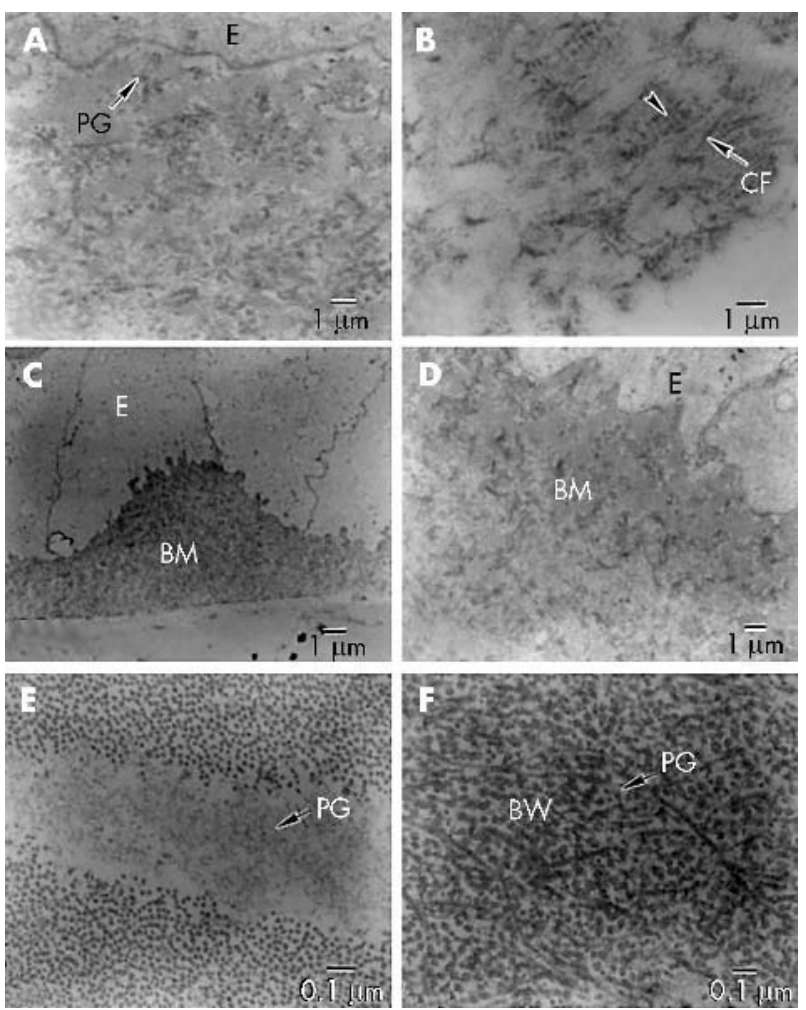

Figure 3 Electron micrograph of subepithelial region affected by mare's tail lines. (A) Proteoglycans (PG) and collagen fibres in basement membrane material below epithelium (E). (B) Long spacing collagen (arrowhead) and collagen fibres (CF). (C) Mounds of basement membrane material (BM) below the epithelium (E). (D) Hemidesmosomes were absent between the basement material (BM) of the mounds and epithelium (E). (E) Proteoglycans (PG) pool in between collagen fibres. (F) Island of Bowman's layer (BW) within an accumulation of basement membrane material. 
sweep of its tapering ends also resembles a "handlebar moustache" (fig 1). ${ }^{5}$ The lines are thickest where they are bunched together, and trail off to become fine toward their ends, where they have some similarity to fingerprint lines. They may occur anywhere on the corneal surface, without preference for the interpalpebral zone, and though they may approach the corneal limbus, they do not reach it. They may be unilateral or bilateral and when bilateral may show some degree of symmetry. They occur in the later decades of life.

Mares tail lines have clinical features in common with fingerprint lines and may sometimes be associated with this. $^{235}$ Clinically, fingerprint lines appear as multiple, curved, concentric lines which are refractile on retroillumination but invisible on focal illumination. Mare's tail lines show a similar parallel arrangement but are less refractile and are best seen in broad, oblique focal illumination. Both disorders are associated with extensive invaginations of the epithelium by basement membrane like material $^{89}$ and each disorder thickens the epithelium locally to cause breaks in the overlying tear film. It is likely that the refractile nature of fingerprint lines relates to that part of the invagination which passes antero posteriorly to enter the epithelium and is imaged end on during retro-illumination. It would appear that, in the case of fingerprint lines, the component which lies in the plane of the epithelium is either too thin, or of a refractive index too close to that of the epithelium too optically homogenous, to scatter light significantly. Conversely, it would appear that the thickness of the invaginations in mare's tail disorder, in combination with the substantial subepithelial accumulations of basal laminar material in this condition, give rise to a greater degree of light scattering and visibility in focal illumination.

Light microscopy in our study was similar in both cases and showed an epithelium thickened by invaginating basement membrane overlying a greatly thickened, connective tissue layer. Ultrastructural study showed the subepithelial accumulations to be multilaminar, an observation also made by Rodrigues et al in relation to fingerprint lines. ${ }^{9}$ The production of a multilaminar, subepithelial basement membrane is well known as a non-specific reaction in a range of unrelated epithelial corneal disorders, ${ }^{9}$ which include Meesmann's corneal dystrophy, ${ }^{11}{ }^{12}$ Fuchs' dystrophy, ${ }^{13}{ }^{14}$ and also the carrier state for Fabry disease. ${ }^{15}$ Rodrigues et al, studying map dot fingerprint disorder, found a subepithelial collagenous zone separating the epithelium from the subjacent basal lamina, whereas we found a more heterogeneous arrangement of irregularly alternating collagenous and basement membrane like material. ${ }^{9}$ It is assumed that, in the epithelial basement membrane disorders, including mare's tail disorder, there is a functional

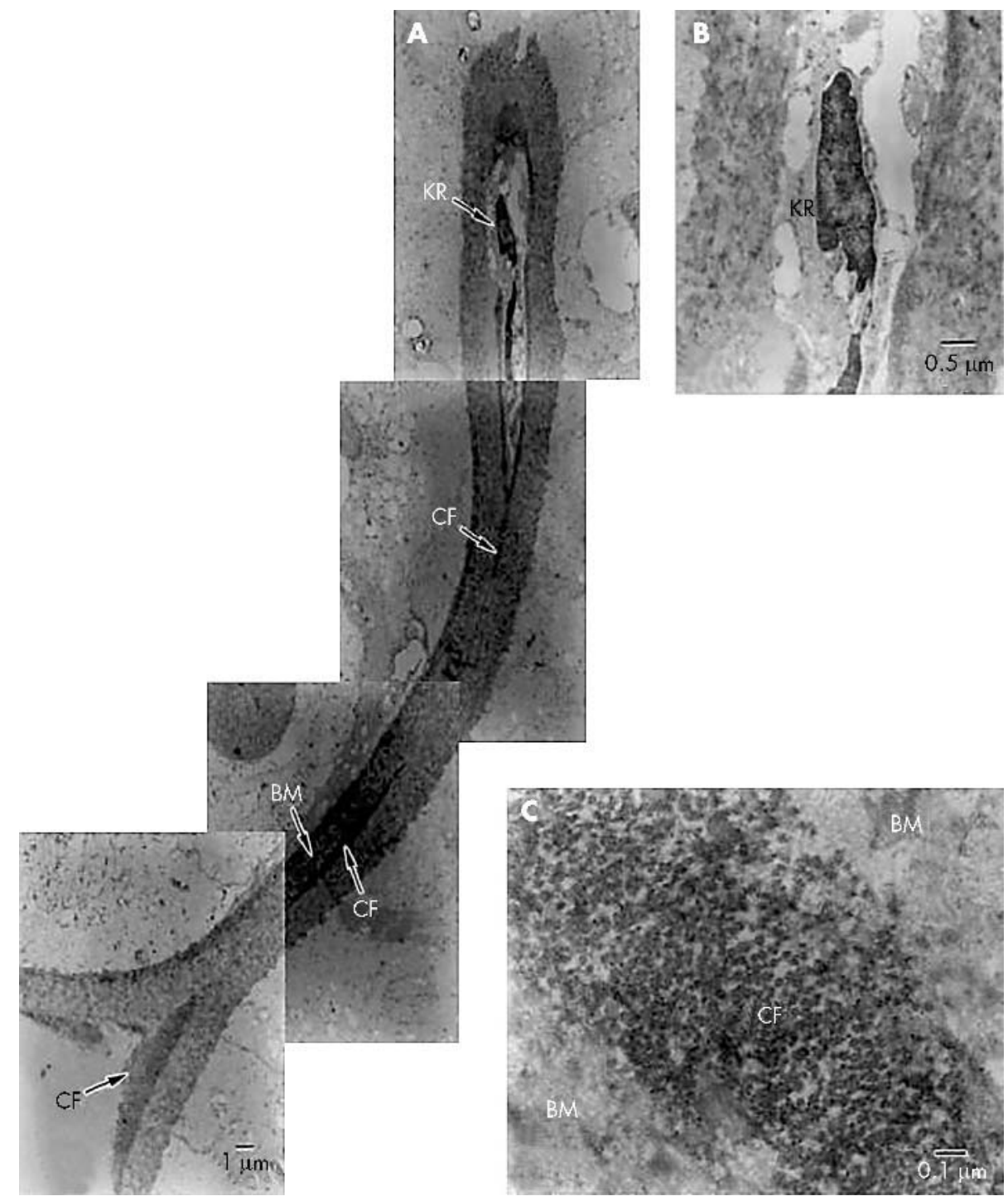

Figure 4 Electron micrograph showing an invagination of the epithelium by basement membrane material. (A) High magnification showing keratocytes (KR), collagen fibres (CF) and proteoglycans. (B) Keratocyte (KR) at the apex of the invagination. (C) Collagen fibres in the middle of invagination. 

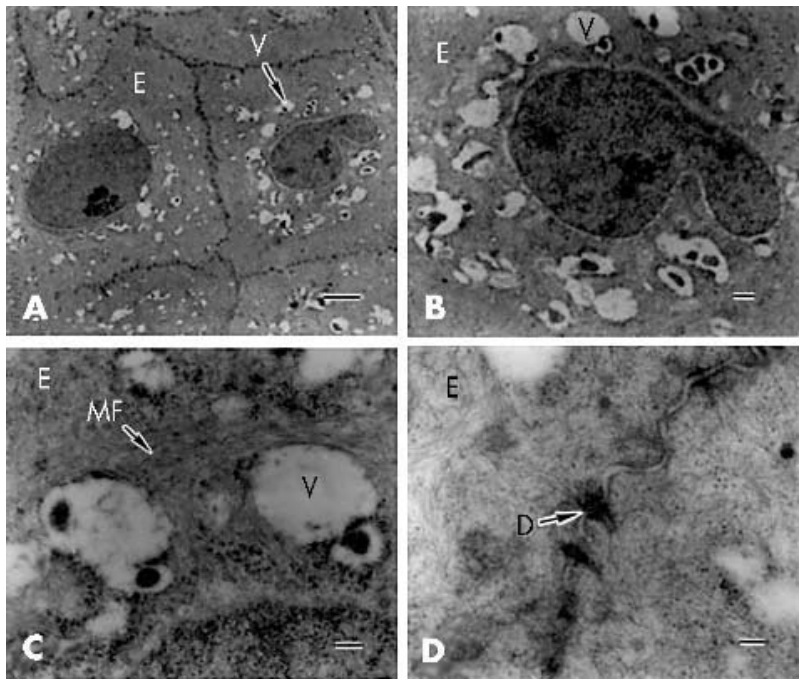

Figure 5 Electron micrograph of epithelium (E) containing mare's lines. ( $A$ and $B$ ) electron dense cytoplasm containing apoptotic nucleus with blebbing and perinuclear vacuoles (V). (C) Large number of microfilaments (MF) around vacuoles (V). (D) Well developed desmosomes (D) in epithelium (E).

abnormality of the epithelial cells, leading to both a disordered production of basement membrane like materials and the deposition of collagen. These give rise to the subepithelial deposits and ultimately to the intraepithelial invaginations. In our study, both the intraepithelial and subepithelial deposits were rich in both proteoglycans and collagen, and in a finely banded $(12.5 \mathrm{~nm})$ fibrillar material, which has also been reported by others. ${ }^{89}$ Although it is assumed that the basement membrane like material is of epithelial origin, the presence of occasional keratocytes suggests that there may be a stromal contribution. It is presumed that both epithelial invagination, and the separation of the epithelium from its normal basement membrane, is facilitated by a faulty assembly of attachment structures, including desmosomes and hemidesmosomes. However, it cannot be excluded that their absence in relation to the abnormal material is a secondary phenomenon. Although a paucity of hemidesmosomes might put patients at risk of recurrent erosion, it is possible that this risk is offset by the basal laminar invaginations, which could serve an anchoring role.

The horizontal disposition of mare's tail lines is distinctly different from that of fingerprint lines. A simple explanation for this difference could be that the invagination process in mare's tail disorder is influenced by the downward, tractional forces imposed by blinking and cause a horizontal draping of the invaginating material.

\section{CONCLUSION}

Mare's tail lines are caused by an invagination of corneal epithelium by basal laminar material. Their visibility in the focal beam of the slit lamp is likely to be because of the thickness and light scattering properties of the invaginations and of the subepithelial deposits. Tractional forces imposed by lid action could explain their draped, horizontal disposition.

\section{Authors' affiliations}

S Akhtar, K M Meek, Department of Optometry and Vision Sciences, Cardiff University, Cardiff, UK

A J Bron, L W Voon, Department of Ophthalmology, University of Oxford, Oxford, UK

Correspondence to: Dr S Akhtar, Department of Optometry and Vision Sciences, Cardiff University, Redwood Building, Kings Edward VII Avenue, PO Box 905, Cardiff CF10 3NB, UK

Accepted 4 November 2003

\section{REFERENCES}

1 Kaufman H, Clower I. Irregularities of Bowman's membrane. Am J Ophthalmol 1966:61:227-30

2 Guerry D. Fingerprint lines in the cornea. Am J Ophthalmol 1950;33:724-6.

3 Cogan D, Donaldson D, Kuwabara T, et al. Microcystic dystrophy of corneal epithelium. Trans Am Ophthalmol Soc 1964;62:213-35.

4 Guerry D. Observations in Cogan's microcystic dystrophy of corneal epithelium. Trans Am Ophthalmol Soc 1965;63:320-34.

5 Brown NA, Bron AJ. Superficial lines and associated disorders of the cornea. Am J Ophthalmol 1976;81:34-51.

6 Laibson PR. Microcystic corneal dystrophy. Trans Am Ophthalmol Soc 1976;74:488-531.

7 Egerer I. Die landkartenartige dystrophic hornhaunt. KlinMbi Augenheilk 1980;177:791-3.

8 Brodrick JD, Dark AJ, Peace GW. Fingerprint dystrophy of cornea. Arch Ophthalmol 1974:92:483-9.

9 Rodrigues MM, Fine SS, Laibson PR, et al. Disorders of the corneal epithelium. A clinicopathologic study of dot, geographic, and fingerprint patterns. Arch Ophthalmol 1974;92:475-82.

10 Laibson PR, Krachmer JH. Familial occurrence of dot (microcystic), map, fingerprint dystrophy of the cornea. Invest Ophthalmol 1975;14:397-9.

11 Kuwabara T, Ciccarelli EC. Meesmann's corneal dystrophy. Arch Ophthalmol 1964;71:676-82.

12 Burn R. Meesmaan's dystrophy. Trans Am Ophthalmol Soc 1968;66:530-635.

13 Schroeder GT, Hanna C. Unusual epithelial changes in a case of combined corneal dystrophy of Fuchs. Am J Ophthalmol 1971;72:542-8.

14 Iwamoto T, De Voe AG. Electron microscopic studies on Fuchs' combined dystrophy: II. Anterior portion of the cornea. Invest Ophthalmol 1971;10:29-40.

15 Weingeist TA, Blodi FC. Fabry's disease: Ocular findings in a female carrier. Arch Ophthalmol 1971;85:169-76. 